\section{Microbial allies against malaria}

Glycans such as $\alpha$-gal expressed by bacteria can elicit specific antibody production, and indeed humans often have high concentrations of such antibodies in their circulation. In Cell, Soares and colleagues show that antibodies to $\alpha$-gal (anti- $\alpha$-gal) can be protective in malaria (infection with Plasmodium species). During the malaria season, Malian children with higher concentrations of anti- $\alpha$-gal have a lower incidence of infection. Indeed, Plasmodium sporozoites have on their surface $\alpha$-gal that can be bound by these antibodies. A mouse model shows that the production of anti- $\alpha-$ gal depends on the presence of the gut microbiota, and specific reconstitution of germ-free mice with bacteria rich in $\alpha$-gal generates anti- $\alpha$-gal protective against Plasmodium infection. Depending on the isotype, anti- $\alpha$-gal protect via complement fixation and/or by opsonization. Protection mediated by anti- $\alpha$-gal seems to operate specifically at the early stages of exposure to Plasmodium by blocking the infection of hepatocytes. Cell 159, 1277-1289 (2014)

\section{Fueling T cells}

$\mathrm{T}$ cells undergo metabolic reprogramming during differentiation, but the mechanistic basis of these changes is incompletely understood. In the Journal of Clinical Investigation, Rathmell and colleagues examine the metabolic requirements of effector cells (the $\mathrm{T}_{\mathrm{H}} 1$ and $\mathrm{T}_{\mathrm{H}} 17$ subsets of helper T cells) and regulatory T cells ( $\mathrm{T}_{\text {reg }}$ cells) in vitro and in vivo. $\mathrm{T}_{\mathrm{H}} 1$ and $\mathrm{T}_{\mathrm{H}} 17$ cells rely on glycolysis, whereas $\mathrm{T}_{\text {reg }}$ cells have low glycolytic capacity and use mitochondrial and fatty acid oxidation. Metabolomic analysis identifies the kinase PDHK1 as serving a key role in metabolic control of the function and survival of specifically $\mathrm{T}_{\mathrm{H}} 17$ cells and $\mathrm{T}_{\text {reg }}$ cells but not $\mathrm{T}_{\mathrm{H}} 1$ cells, with high, intermediate and low expression of PDHK1, respectively, by these cells. Blockade of PDHK1 in mouse models of inflammatory bowel disease or experimental allergic encephalitis both increases the number of $\mathrm{T}_{\text {reg }}$ cells and diminishes that of $\mathrm{T}_{\mathrm{H}} 17$ cells in vivo. PDHK1 therefore sits at a key signaling juncture that controls the glycolytic and oxidative metabolism of T cells.

ZF

J. Clin. Invest. (1 December 2014) doi:10.1172/JCI76012

\section{Platelet checkpoint}

Neutrophils in inflamed blood vessels adopt a polarized morphology and begin crawling on endothelial cells before their extravasation into the tissues. In Science, Hidalgo and colleagues describe interactions between activated platelets and neutrophils that provide a checkpoint for neutrophilic function during inflammation. Polarized neutrophils cluster the P-selectin ligand PSGL-1 on their uropods, which causes platelet accumulation at their trailing edge. Blocking this interaction blunts the polarization of other receptors on neutrophils, including the integrin Mac-1 and the chemokine receptor CXCR2, and alters their crawling activity. Notably, loss of the interaction of platelets with luminal neutrophils also blocks the release of neutrophil nets and lessens tissue damage in several models of inflammation. Thus, neutrophils seem to utilize activated platelets as a 'go' signal to enter inflamed tissues.

Science 346, 1234-1238 (2014)

\section{$L A D$}

\section{Unfold and destroy}

Defensins are short cysteine-rich peptides that can inactivate bacterial toxins, but the mechanism by which they induce such inactivation has remained unclear. In Immunity, Kudryashova et al. show that the human neutrophil $\alpha$-defensin HNP1 induces the unfolding of bacterial toxins, which results in the exposure of hydrophobic protein regions and instability, enhanced susceptibility to proteolysis, precipitation and functional inactivation. HNP1 inhibits the normal autoprocessing and increases the susceptibility of susceptible toxins, such as the Martx toxin from Vibrio cholerae, to proteolysis, but not that of mammalian enzymes or non-susceptible bacterial toxins. Binding of HNP1 to Martx induces unfolding and conformational changes in the tertiary and secondary structure of the toxin but not that of the defensin. These effects operate at normal physiological concentrations of salt and defensins and are shared by other defensins assessed, which suggests they represent a shared mechanism.

Immunity 41, 709-721 (2014)

\section{The fetal imprint of tissues}

The contribution of fetal macrophages, relative to that of macrophages derived from hematopoietic stem cells (HSCs), to adult tissues remains unclear. In Nature, Rodewald, Geissmann and colleagues show that most adult tissue macrophages originate from yolk sack-derived erythro-myeloid progenitors (EMPs) that are distinct from HSCs. Tracing of fetal and adult HSCs with the marker Flt3 shows that only $14 \%$ of liver Kupffer cells, $2 \%$ of brain microglia, $30 \%$ of epidermal Langerhans cells and $40 \%$ of lung alveolar macrophage are HSC derived in 1-year-old mice. Pulse labeling for expression of the endothelial cell receptor Tie2 shows that yolk sack-derived EMPs generate all fetal tissue macrophages up to embryonic day $\mathbf{1 5 . 5}$ of mouse development and also contribute to $30-60 \%$ of tissue-resident macrophages in adult mice. Yolk sack-derived macrophages are replaced by HSC-derived cells only marginally in the brain, liver and epidermis of adult mice at steady state. Populations of yolk sack-derived EMPs expand in the fetal liver, where they overlap with Flt3 ${ }^{+}$HSC-derived progenitors; this suggests caution should be taken in the interpretation of experiments using fetal liver progenitor cells. IV Nature (3 December 2014) doi:10.1038/nature13989

\section{Balancing protein degradation}

Cells degrade proteins via cytosolic proteasomes or vesicular lysosomes, followed by further processing by peptidases to allow recycling of amino acids. In Cell, Lu et al. identify several kindreds associated with genetic deficiency in the expression of tripeptidyl peptidase II (TPPII). Patients present with multiple immunological deficiencies and autoimmunity and encounter recurrent bacterial and viral infections. TPPII functions downstream of proteasomes. Loss of TPPII alters amino acid homeostasis in cells, which triggers a compensatory increase in lysosomal protein-degradation pathways. However, increased lysosomal activity leads to further defects in cellular metabolism by catabolism of the key glycolytic enzyme hexokinase-2, which decreases the glycolytic capacity of the affected cells. As glycolysis is required for many functions of activated cells of the immune system, loss of TPPII activity severely affects the proliferation and effector function of these cells, as observed in the affected patients.

$L A D$

Cell 159, 1578-1590 (2014) 